\title{
Noise sensitivities in dogs: a new licenced treatment option
}

Kevin McPeake ${ }^{a}$, Affenzeller Nadja ${ }^{\text {a,b }}$, Daniel Mills ${ }^{a}$.

a Animal Behaviour, Cognition and Welfare Group, School of Life Sciences, University of Lincoln, Green Lane, Lincoln, LN6 7DL, United Kingdom

${ }^{b}$ Department of Companion Animals, Clinical Unit of Internal Medicine Small Animals, University of Veterinary Medicine Vienna, Veterinärplatz 1, 1210 Vienna, Austria

Corresponding author: Kevin McPeake

kmcpeake@lincoln.ac.uk

Noise sensitivities, i.e. fear, anxiety and phobia based responses to noise/sound stimuli (Sherman and Mills 2008), have a high prevalence in dogs: Blackwell and others (2013) found that $25 \%$ of owners surveyed in the UK reported their dog to be fearful of noises, with $49 \%$ of owners in the same study indicating that their dog displays at least one sign of fear when exposed to loud noises, which is consistent with the $42 \%$ reported in the 2015 PDSA Paw report (PDSA 2015). The most commonly reported noise stimulus triggering a response in these studies is fireworks, but this problem is more than a seasonal issue with dogs commonly reacting to a range of other noises such as thunder, gunshots, vehicle and household noises, by showing signs including panting, escape attempts, hiding and destructiveness (Sherman and Mills 2008).

Despite there being well-established treatment programmes for the long-term elimination of the problem (Levine and others 2007), it is a cause for concern that less than a third of owners have sought help for their dog, and the majority of those who do, turn to someone other than their veterinarian (Blackwell and others 2013) suggesting that a large number of dogs remain untreated for noise sensitivities. Underlying medical problems reported to be associated with the onset of a noise sensitivity in dogs include cognitive dysfunction (Landsberg and others 2011), suboptimal thyroid function (Aronson and Dodds 2005; Dodman and others 2013) and, based on our own experience, a range of pain- 
related problems. The veterinary profession should be at the forefront of actively promoting the potential to solve the problem of noise sensitivities in dogs.

Long term management involves desensitising the dog to the fear-provoking noise over an extended time period and although time consuming has an excellent prognosis with the right treatment programme; over $90 \%$ of owners completing an 8 week training programme using Adaptil ${ }^{\mathrm{TM}}$ pheromone diffuser alongside a sound CD reported improvement (Levine and others 2007). One of the CDs was the Sounds Scary ${ }^{\mathrm{TM}}$ programme, now available free of charge from https://www.dogstrust.org.uk/help-advice/dog-behaviour-health/soundtherapy-for-pets .

Many owners will try to avoid or minimise the impact of the problem by, e.g. walking in daylight hours, shutting windows/doors, playing the radio/television/music to mask the noise. If the problem is severe, then they may seek help from their vet, but often shortly before noise exposure e.g. Guy Fawkes Night or New Year's Eve. In this situation there is no time to implement effective training to desensitise the dog and so there is a need for short term management interventions that will help reduce the dog's suffering at this time.

In these circumstances there is undoubtedly a role for short acting medication with a rapid onset that alleviates the fear and anxiety in the short term. The use of benzodiazepines e.g. alprazolam (Crowell-Davis and others 2003), and diazepam (Herron and others 2008), and the alpha-2 adrenergic receptor agonist clonidine (Ogata and Dodman 2011) have been recommended for treatment, but these are not licensed, and their efficacy in our experience is quite variable. In the current issue of the Veterinary Record, Korpivaara and others (2017) examine the efficacy of a new licensed formulation of the alpha-2 adrenoreceptor agonist dexmedetomidine administered at $0.1 \mathrm{mg} / \mathrm{ml}$ as an oromucosal gel against a placebo in alleviating clinical signs of anxiety and fear associated with fireworks in dogs. A higher proportion of dogs were reported by their owners as having an excellent or good overall treatment effect (compared to what they recalled from the previous year) in the dexmedetomidine oromucosal gel group (72\%) compared to the placebo group (37\%). In addition, owners were asked to score clinical signs and extent of anxiety and fear on a 
scale of 12 behavioural signs: panting, trembling, vocalising, pacing and house soiling were 5 of the 12 behavioural signs found to be significantly lower (suggesting less distress) in the dexmedetomidine group when compared to the placebo group. Owners assessed functional alertness by calling their dog and scoring their observations (related to responsiveness and ability to stand and walk) with $>85 \%$ of dogs being classed as fully functional with no apparent differences between groups demonstrating that dexmedetomidine appeared to be at a sub-sedative dose for the majority of dogs.

Studies like these are important because they provide the veterinary field with a stronger evidence base for new potential products in a market that has a wealth of largely untested supplements and suggestions, which may be far less useful. Indeed as noted in this study and elsewhere (Cracknell and Mills 2008), there is a large placebo effect to many treatments for behaviour problems, and so veterinarians need to be careful not to be seduced by untested alternatives. They also need to comply with the prescription cascade when considering the use of medication, and so need to be aware that dexmedetomidine oromucosal gel (Sileo ${ }^{\mathrm{TM}}$, Zoetis) is currently the only product approved by the European Medicinal Agency and Food and Drug Administration for treating canine noise aversion associated with fear and anxiety.

The study also highlights the value of finding better ways of administering effective treatments to ensure what works in theory also works in practice. The gel is applied onto the oral mucosa of the dog's cheek and the vast majority of owners (84.6\%) found the product easy to administer. However it is worth noting that in dogs that are not accustomed to handling procedures administration has been reported to be more difficult and so there is in an important role for the veterinary team to give advice on how to teach a dog to accept such handling.

However, it is important for veterinarians to recognise that their responsibility does not end with the dispensing of medication. It is important that the veterinary team is pro-active in following up such cases when the event is over, to ensure that the long term problem and root cause of the issue is addressed. Out of season group desensitisation classes are an efficient way of helping and 
caring for a large number of clients, who can be taught through the principles of a generic programme and form a support group to aid motivation (Sherman and Mills 2008). Many veterinarians feel ill-prepared to manage behaviour problems in practice (Roshier and McBride 2013) but they do not need to have specialist training to offer a professional service when it comes to managing noise fears. As veterinarians, they need to evaluate the health of the animal and ensure the problem is not associated with some underlying medical problem; they are also responsible for any medication they prescribe and need to understand the indications and contraindications and when (and to whom) to refer for specialist help, but other members of the team can take on the wider client education and training roles, and many will do so enthusiastically. In this way all will benefit.

This new product may also have a wider range of applications for which there is currently no licensed product. This includes other situationally occurring fears and anxieties such as fear of the veterinary clinic and associated handling (see papers by Hopfensperger and others (2013) and Cohen and Bennett (2015) who used oromucosal detomidine gel and dexmedetomidine injectable administered orotransmucosally respectively, to facilitate handling of dogs in the veterinary context at sedative doses).

In the authors' experience, many dogs with fear of fireworks develop an overall increase in anxiety around the time of year with some dogs not wanting to leave the house despite no audible fireworks. In addition owners also report mood changes that are most likely due to repeat exposure to anxiogenic stimuli and/or anticipation of noises occurring. For other dogs, noise sensitivity to fireworks may represent only one of many noises which trigger fear and anxiety all year round, and can be only one of several expressions of an underlying fearful/anxious temperament, such as social fears/anxieties, separation related problems and generalised anxiety disorders. The optimum treatment of such cases may differ to those acute and occasional noise fears, and may include the use of long term psychoactive medication e.g. clomipramine (Crowell-Davis and others 2003) or fluoxetine (Ibáñez and Anzola 2009) potentially alongside a short acting anxiolytic, such as dexmedetomidine or a benzodiazepine as needed, and this combination should be safe, assuming the animal is in good physical health. 
Medication(s) chosen should always be tailored to the individual based on a thorough review of the behavioural history (screening for concurrent fears and anxieties) and medical history (screen for underlying disease, potential drug interactions or contraindications) and should always be provided alongside management advice and a behaviour modification program that is feasible for both the patients and their caretaker within the daily constraints of everyday life.

In conclusion, dexmedetomidine gel, provides an important part of the arsenal of any welfare centred practice and extends the opportunity for veterinarians to more fully engage with the prevalent welfare problem of noise sensitivity.

\section{Competing interests}

$\mathrm{KM}$ has previously conducted research funded by Boehringer Ingelheim. DM is employed as a consultant to Ceva Animal Health and Orion mentioned in this article and undertakes consultancy for a number of other companies interested in the production of behaviour modification products. NA has no competing interests to declare.

\section{References}

ARONSON, L. P. \& DODDS, W. J. (2005) The effect of hypothyroid function on canine behavior. Proc. Int. Vet. Beh. Med, 228

BLACKWELL, E. J., BRADSHAW, J. W. \& CASEY, R. A. (2013) Fear responses to noises in domestic dogs: Prevalence, risk factors and co-occurrence with other fear related behaviour. Applied Animal Behaviour Science 145, 15-25

COHEN, A. E. \& BENNETT, S. L. (2015) Oral transmucosal administration of dexmedetomidine for sedation in 4 dogs. The Canadian Veterinary Journal 56, 1144

CRACKNELL, N. R. \& MILLS, D. S. (2008) A double-blind placebo-controlled study into the efficacy of a homeopathic remedy for fear of firework noises in the dog (Canis familiaris). The Veterinary Journal $177,80-88$

CROWELL-DAVIS, S. L., SEIBERT, L. M., SUNG, W., PARTHASARATHY, V. \& CURTIS, T. M. (2003) Use of clomipramine, alprazolam, and behavior modification for treatment of storm phobia in dogs. Journal of the American Veterinary Medical Association 222, 744-748

DODMAN, N. H., ARONSON, L., COTTAM, N. \& DODDS, J. W. (2013) The effect of thyroid replacement in dogs with suboptimal thyroid function on owner-directed aggression: $A$ randomized, double-blind, placebo-controlled clinical trial. Journal of Veterinary Behavior: Clinical Applications and Research 8, 225-230

HERRON, M. E., SHOFER, F. S. \& REISNER, I. R. (2008) Retrospective evaluation of the effects of diazepam in dogs with anxiety-related behavior problems. Journal of the American Veterinary Medical Association 233, 1420-1424 
HOPFENSPERGER, M. J., MESSENGER, K. M., PAPICH, M. G. \& SHERMAN, B. L. (2013) The use of oral transmucosal detomidine hydrochloride gel to facilitate handling in dogs. Journal of Veterinary Behavior: Clinical Applications and Research 8, 114-123

IBÁÑEZ, M. \& ANZOLA, B. (2009) Use of fluoxetine, diazepam, and behavior modification as therapy for treatment of anxiety-related disorders in dogs. Journal of Veterinary Behavior: Clinical Applications and Research 4, 223-229

KORPIVAARA, M., LAAPAS, K., HUHTINEN, M., SCHÖNING, B. \& OVERALL, K. (2017)

Dexmedetomidine oromucosal gel for noise-associated acute anxiety and fear in dogs-a randomised, double-blind, placebo-controlled clinical study. Veterinary Record, vetrec-2016104045

LANDSBERG, G. M., DEPORTER, T. \& ARAUJO, J. A. (2011) Clinical signs and management of anxiety, sleeplessness, and cognitive dysfunction in the senior pet. Veterinary Clinics of North America: Small Animal Practice 41, 565-590

LEVINE, E. D., RAMOS, D. \& MILLS, D. S. (2007) A prospective study of two self-help CD based desensitization and counter-conditioning programmes with the use of Dog Appeasing Pheromone for the treatment of firework fears in dogs (Canis familiaris). Applied Animal Behaviour Science 105, 311-329

OGATA, N. \& DODMAN, N. H. (2011) The use of clonidine in the treatment of fear-based behavior problems in dogs: an open trial. Journal of Veterinary Behavior: Clinical Applications and Research 6, 130-137

PDSA (2015) PDSA animal wellbeing (PAW) report.

https://www.pdsa.org.uk/ /media/pdsa/files/pdfs/veterinary/paw-reports/pdsa-paw-report2015.ashx?la=en. Accessed 25 March, 2017

ROSHIER, A. \& MCBRIDE, E. (2013) Veterinarians' perceptions of behaviour support in smallanimal practice. Veterinary Record 172, 267-267

SHERMAN, B. L. \& MILLS, D. S. (2008) Canine anxieties and phobias: an update on separation anxiety and noise aversions. Veterinary Clinics of North America: Small Animal Practice 38, 1081-1106 